\title{
Theoretical perspectives upon the return to work of cancer patients: The difficult path of integration in the organization
}

\author{
Radu-Ioan Popa ${ }^{1 *}$ \\ ${ }^{1}$ Lucian Blaga University of Sibiu, Centre for Social Research, 2A Lucian Blaga Street, 550169 - Sibiu, Romania
}

KEYWORDS

Cancer patients

Return to work

Integration

\begin{abstract}
The present article follows an in-depth analysis of several relevant articles and major findings concerning the return to work of cancer patients, in various situations, from a manager and patient point of view, putting into discussion the effects and consequences of different factors that may influence the well-being of the patient at work and impact the organizational life. The concepts of returning to work and integration are scarcely analysed throughout the scholarly literature in the case of employees diagnosed with cancer, due to several reasons presented in the paper: from the complex topic of investigation that many studies fail to approach in terms of confidentiality, technical, ethical and moral grounds to the specific and difficult apparatus for research in the case of an even more complex, multiple instances and personalized manifestation long-term illness. In conclusion, the general framework solicits for a more integrated model of research and future multi-facet schemes for interventions, considering that there is a general consensus focusing on the need for connecting the health services with the employee and employer level, alongside stakeholders' active participation.
\end{abstract}

\section{Introduction}

The present paper explores the today's challenges of the organizations when facing the return to work (RTW) of cancer patients, focusing here on

\footnotetext{
*Contact address: radu.popa@ulbsibiu.ro (Radu-Ioan Popa)
} 
the managers' and patients' perspectives, all related to the different issues of integration.

Integration as a complex social process for any employee imposes a certain amount of time, adequate conditions, acceptance from the other colleagues and of course a certain psychological state for involvement and active participation.

From another point of view, integration can be seen as a two facet dimension, one following the professional or technical integration of the employee, who needs a certain background concerning education, skills or competences to fulfil the job demands while the other focuses on the psychosocial domain, the so-called 'in-depth level' which describes the employee's adherence to the organization's values, own identity and social status and role inside the organization, also team role and position, creating and developing specific attitudes towards work, job position, colleagues and leadership, etc.

Now, in the case of former patient employees, the process of integration solicits an even more complex and specific long-term itinerary. In this light of things, specific for cancer patients, the studies of Spelten, Sprangers and Verbeek (2002) and Steiner et al. (2004) described several factors or variables that should be taken into consideration when focusing on the return to work of such employees such as (1) health variables: disease stage, cancer type, time since treatment, physical symptoms, and fatigue; (2) work-related variables: positive attitudes of co-workers, control over work hours, manual labour, and physical demands at work; (3) psychosocial variables: social support, attitudes towards the value of work, team role, discrimination effect, etc.

In many cases, the scholarly literature includes research articles that focus on finding a specific model for evaluation, prevention and intervention for this long-term challenge, considered by many a true disability that affects the well-being of the worker-former patient and the entire organization (Feuerstein et al. 2010). For example, Feuerstein et al. $(2010,416-417)$ presented in the above-mentioned article that out of a review 
of 100 potential papers on RTW interventions for female breast cancer survivors, only 4 studies focused on rigorous inclusion criteria and of these 4 , only a single study used a control group. From this perspective, inclusion being such a complex and multi-facet dimension, it is analysed by a heterogeneous collection of articles that evaluate in a distinct manner elements of RTW and the association with inclusion.

Moreover cancer can be seen as a severe, complex and long-term illness, with multiple forms and symptoms, which imports a detailed attention and analysis when researched. From this point of view any research attempt becomes difficult especially when it comes to study the illness in a unitary design, generalising findings and testing result validity. Any scholarly debate and research article will have to choose, when targeting the issue, a certain type of cancer, a specific patient study group, a strict set of variables for various experimental associations, many a time the limitations and co-variables affecting the study results.

The research interest in cancer and RTW is without any doubt increasing, the topic being closely related to the medical improvements in treatment and medical discoveries in recent years, together with the process of aging, active aging process and the strategies for social inclusion inside the organization. From this perspective, the patient diagnosed with cancer improves his or her chances for survival due to medical new treatments and discoveries; he or she finds better conditions for RTW due to employer attention and involvement; he or she gains more opportunities for hiring, maintaining a job and changing jobs due to employers, policy makers and stakeholders' active involvement in creating and developing social inclusion strategies and policies.

Nowrouzi et al. (2009) stated in their research paper that cancer has as well an individualistic image, as mentioned before, which makes it difficult to investigate and analyse; it is an illness that affects the multiple levels of the employee: the health status of the patient, the quality of subject's life, the social network, the support network, the health and safety 
legislation, the employment and integration mechanisms and of course performance, motivation and productivity at work.

\section{General debate upon variables, cancer and RTW}

First of all, considering the health variables and following the research literature (Spelten, Sprangers and Verbeek 2002; Steiner et al. 2004), cancer patients can be divided into: former patients that are RTW, patients with cancer that are trying to maintain their work or RTW, patients that are in remission and RTW, patients that fully recovered from cancer but remain with long-term collateral disabilities and RTW. All the presented categories will solicit from the employer a great deal of attention and work adjustment. From this point of view, in their paper Munir, Yarker and McDermott (2009) described the importance and influence of employer support and work adjustment on the work ability of those patients following treatment and also coming to work. Their literature review on the topic also pointed out that there is a need for more longitudinal studies on the matter, which could better underline the impact and effect of factors such as: treatment response, side-effects of illness, degree of fatigue and re-occurrence of cancer, gender variable, etc.; all of these factors having a direct association with the work ability, re-setting of work space and conditions and offering a proper support from the employer (Munir, Yarker and McDermott 2009).

Now, in the case of the first category of former patients, who are returning to work, completing treatment, the major challenge consists of integrating them in a new fully operational team or position on the worksite. Following absence this can lead, in many cases, to a severe decrease in motivation level, low performance, longer periods of time for adaptation, in some cases losing track of working technologies and expertise, difficulties in task management and re-establishing professional networks while ensuring an effective integration to the team, and not outside of it. For example, in a research study, Lavigne et al. (2008) confirmed that breast cancer survivors were 3\% lower than the healthy control group in work productivity. The 
authors compared the stage 0 , stage 1 and 2 breast cancer survivors and found correlations with the reductions in work performance even after three years post treatment (Lavigne et al. 2008). On a target group in another organizational culture and environment in Finland, Taskila et al. (2007) discovered that, among cancer survivors, their physical work ability had deteriorated with $26 \%$ and mental work ability decreased with $19 \%$ due to cancer.

Overall, in this situation the employer has the best chance to integrate the subject, who may be fully restored to a work capacity that only needs a small amount time and support in order to adapt, integrate, perform and obtain results in the organization.

In the second category, we find the patients with several levels of cancer that are trying to maintain their work or RTW. The list of situations is wide and complex: some of the patients are forced to change jobs for appropriate health and work conditions, others are struggling to RTW after several work interruption periods in order to follow treatment, some patients strive to maintain their job, keep up with the work and demands while following treatment and illness manifestation. In a longitudinal study conducted by Park, Park and Kim (2009) on a target group from organizations in South Korea, it has been concluded that cancer patients were more likely to lose their job than cancer-free individuals. The same authors also suggested that in terms of illness type patients with pancreatic, lung, brain, central nervous system cancer or non-Hodgkin's lymphoma were more likely to lose their jobs sooner than other cancer patients, due to the severity and aggressive development of the illness (Park, Park and Kim 2009). In the same direction, Syse, Tretli and Kravdal (2008), found on another organizational target population in Norway that leukemia, nonHodgkin disease, brain, bone, lung, colorectal, and head-and-neck cancer were associated with lowered employment for both genders (ranging from $25 \%$ to $80 \%$ ), compared to the general population.

After Munir, Yarker and McDermott (2009), the occupational health professionals can play a vital role in the employer's difficult task to manage 
and integrate the employee diagnosed with cancer in the organization's functional structure. The management team needs to constantly monitor the health status of such employees, to assess and evaluate their capacity, to readjust work conditions and environment, to maintain a direct contact with other social actors, such as the family support group, the medical team and of course the employee, following closely the progress of the illness. In this manner, the management's main concern will be the employee's well-being at work and not their work ability, performance being replaced by motivation appraisal, well-being, inclusion and support, active participation in the healing process if possible. On the opposite side, the organization provides data that sustain the fact that such employees cost in terms of time, effort and finance the company that many a time will try to renounce swiftly, avoiding if possible any involvement in work adjustment and support on the long run (Amir et al. 2010). Park, Park and Kim (2009) also discovered that the amount of time before re-employment was significantly longer in cancer patients than in the control group and that the subjects face a difficult barrier when it comes to re-hiring, especially if previously diagnosed with pancreatic, lung, brain, central nervous system cancer or nonHodgkin's lymphoma.

The third category of patients consists of those subjects who were previously diagnosed with cancer, who followed and still are following treatment, being in remission and are RTW. Now, we must mention that the term in remission does not mean as some may misinterpret, that the patient is fully recovered. The medical concept itself refers to a health state of the patient that is improving, underlining that he or she is responsive to a certain treatment, and that the health conditions are stable and that the illness has stopped advancing for a certain period of time. Of course, the remission phase may lead to a complete recovery in some cases, but this is due to many factors from the application of treatments, subject response, time, side-effects to the medical evolution and health status of the employee, which could be unpredictable along the way. In this case, the management team is bound also to a constant monitoring and work adjustment for the 
employee care, following the motivational level, well-being, work ability, degree of integration and communication as well as the constant need for adjusting the work setting and goals for this type of employee.

The final category of patients refers to subjects that surpassed cancer but remained with long-term collateral disabilities and are RTW. In this case, research articles focus on the work conditions of the employee, from the simple location setting (e.g. placement and type of office furniture, light, air, communication means, etc.) to the more complex means of logistics (e.g. application use and access, computer management, tool use, etc.), missing out sometimes that besides the technical integration process there is a greater need for psychosocial integration (Feuerstein et al. 2010).

From this perspective, the management team is bound to reassure the employee's need for communication, for being part of a team, for rebuilding professional networks and receiving acceptance from other colleagues and support. For example, if an employee, after a long term illness involving bone cancer, is left unable to walk, he or she will need adequate means to access the work site and office in a secure manner, will need a personalized working schedule (e.g. reduced working hours, supplementary pauses, postintervention treatment facilities) in order to obtain performance at work in terms of time and quality criteria but also he or she will need to be accepted by the former team or fellow employees, from the disability and work ability perspective.

Concerning the work-related variables, Feuerstein et al. (2010, 431) advance the idea of work environment impact over employees with cancer. The authors include in this setting dimensions that could affect for better or for the worse the entire well-being of such employees, among which: employee perception on job demands and work, organizational and social climate, support from fellow workers and attitudes, job stress, management of tasks and working hours etc. (Feuerstein et al. 2010). From this perspective, we may state that the management system can provide a series of measures starting right from the problem identification and dilemma solving inside the organization concerning a better platform for intervention 
and integration of actual or former cancer diagnosed employees. For example, for any given organization, if the management team or persons in charge of the strategy for integrating employees after RTW, will observe the support of team members for the employee reaches low levels of involvement, should take into consideration a in-depth analysis or team diagnosis to establish the causes. These may range from scarce information on the illness and treatment, misunderstanding of the so-called 'special program' for employees diagnosed with cancer, that in many cases occupy the same position as the other fellow workers, up to the point that such employees act and work in a different manner, sometimes in a slower work rhythm, with a continuous need of support and attention from others. Secondly, following the analysis results, the management team has the opportunity to transform the identified problem in a measure for improving the work related variable for employees diagnosed with cancer. In the above mentioned example, fellow workers or team members can be informed and debriefed in detail about the condition, treatment, measures and requirements involved in this type of illness, raising awareness on the issue and a better understanding of the employee's special case. Moreover, the employees can be informed about the differences in the working schedule and duties for this type of work. Finally, employees may be debriefed on the working style, reactions, needs and demands of cancer patients that RTW. In other cases, team members or fellow employees can be organized in small training groups, short-term sessions, in which they can be motivated and prepared to offer support for those with special needs that are RTW or trying to maintain a job inside the organization. In this manner, the management team can ensure a greater degree of not only understanding, but also active participation and support from employees towards the employee with cancer, developing an in-depth strategy for an optimal integration.

In the case of psychosocial variables, the scholarly literature mentions a wide range of dimensions to be taken into consideration when dealing with the RTW of cancer patients (Spelten, Sprangers and Verbeek 2002; 
Steiner et al. 2004). Among these we can mention: the social support, the attitudes towards the value of work, team role management, the well known discrimination effects and processes and so on.

In a research paper, Amir et al. (2010, 438-441) discovered, for example, five multidimensional factors that are present in the case of line managers attitudes' towards employees with cancer diagnosis in the UK, as follows: a supportive attitude, the tendency to maintain normality, a fearful attitude, the feeling of burden and last but not least the financial factors. Moreover, the study promotes the idea that by using positive attitudes towards patients that RTW, direct managers impact a stronger retention of such employees and ensure a faster integration and adaptation to work.

Now, when it comes to attitudes, it would seem that not only managers, supervisors, decision-making persons or team leaders' position would matter in the case of employees diagnosed with cancer, but also the colleagues or co-workers of the subject, who in various organizational situations may impact more on the employee's well-being, recovery process, performance at work and of course integration. It is important that coworkers are debriefed about the situation of their colleague and urged to offer support for the subject in discussion, while at same time keeping an optimal balance so that he or she would not feel constrained in any way. In some cases, the employee diagnosed with cancer may feel closely monitored, under a certain social pressure or even living the special needs person role, especially when everyone else gives care and attention in an extreme way, from family members to co-workers and managers. The subject in discussion may need a certain independence and self-management at work, to prove that he or she can perform the solicited duties. Amir et al. (2010) described the importance of reaching a state of normality when referring to employees with cancer diagnosis, to integrate such cases under the terms of normality, giving them the chance to evolve, work, develop and make a difference for themselves and others, even if they possess this special state.

From this psychosocial perspective, the patient can relate much more easily to the job, team and organization, feeling involved, requested and 
needed in an environment that offers support and asks for active participation.

\section{Management perspective over cancer and RTW}

As we discussed before, one of the major directions for research on the path of integrating cancer patients at work, consists of evaluating managers' and team leaders' perceptions and attitudes towards cancer and RTW. Grunfeld, Low and Cooper $(2010,615)$ pointed out that there is a tendency among management staff or employers to adopt a negative belief concerning cancer and RTW. The authors suggested key factors and motives that should be taken into consideration when analysing in future studies the present issue, such as: the potential difference between the employee's own expectations concerning the personal abilities and the actual ability to perform a certain task, the fact that managers tend to perceive employees diagnosed with cancer as a source for extra effort and special demands when dealt with, considering the integration process a difficult one and sometimes not needed, raising awareness and acceptance of individual differences were seen also as tools for a better integration of the cancer patient (Grunfeld, Low and Cooper 2010).

In another study, Maiwald et al. (2013, 483-494) presented that managers in a Canadian setting are facing unclear RTW policies and procedures concerning patients, this situation leading to a decrease in managers' active participation for the reintegration of this category of employees. Much more, the authors suggest that there is a general feeling among those managers that were evaluated, for feeling unheard, frustrated and under pressure when dealing with policies and policy execution. In their opinion this situation led to a low involvement in RTW issues and a weak communication with the stakeholders (Maiwald et al. 2013). At the same time, in an Australian organizational setting, McKay, Knott and Delfabbro (2013) found also there is a lack of knowledge among managers concerning the procedures and attitudes imposed by RTW and cancer 
patients. Moreover Hoefsmit, de Rijk and Houkes (2013) observed that even if the legislation supports the idea of cooperation between employer and employee in the case of RTW and illness recovery, there are low levels of information, knowledge, trust and motivation to cooperate among the two sides.

As discussed before, there is a need for participation from all categories of people involved in supporting the RTW of employees with health problems, focusing here on occupational health specialists, health services, employers, co-workers and, of course, stakeholders, in order to avoid low involvement or overcharging one category of social actors while the others are left behind, to ensure a better understanding of policies and health issues.

From another perspective, Mak, Ho and Kim $(2014,726)$ analysed the results in a research conducted upon an organizational setting in Singapore, and stated that employers who participate in integrating cancer survivors in the organization are influenced by factors such as their own efficacy and personal attitude on cancer survivors, followed by employment situation, attitudes towards cancer and perceived moral obligation. In this case, the employer's attitude as a major variable still reaches out, underlining the importance of perception and knowledge upon the illness in discussion for strategy, intervention and effect making. Other studies suggest an approach that takes into consideration the intervention upon the cognitive representations of illness in the case of managers (Grunfeld, Low and Cooper 2010, 616; Nowrouzi et al. 2009, 18). The general idea is that when employers and other co-workers face a colleague or a new employee returning to work after a cancer diagnosis, in the absence of information and perception training, more likely negative perceptions, representations and scenarios will appear. From the cognitive psychology point of view, negative representations upon illness may be changed, through proper information at work and awareness, developing them into accurate cognitive representations, with a positive meaning, which in response may generate pro-active behaviour and supportive attitudes. 
Grunfeld, Low and Cooper $(2010,616)$ presented also that, in general, management should analyse correctly the predictive value of RTW and also other representations and beliefs of the employee with cancer diagnosis. Integration and later on performance starts from the very simple attitude, motives, level of energy and will to involve in work of the employee. So why not work with that first in order to ensure that the person will maintain a job for a longer time?

In the case of patients with cancer that RTW, the first approach that should be taken into consideration when managing integration is perception - the perception of the decision making sector, the perception of co-workers, the perception of partners and external stakeholders, the perception of the employee's family and social support network in order to provide an adequate and individualistic strategy for integration in the second stage of the process. The same authors support the idea of managing phased returns or for the management team to know them at least, a mechanism that functions closely with another procedure that promotes the gradual reintroduction of duties for the patients with cancer (Grunfeld, Low and Cooper 2010). One of the many challenges the persons that RTW face, after a cancer diagnosis, resides in the turnover phenomenon, which affects the individual and the organization as well. The cancer patient, due to many factors such as a complex multi-step treatment, the constant probability for cancer reoccurrence, the irregular fluctuation in work energy, resting periods and fatigue, is bound to quit the designated tasks, job, role and organization much easier than the average worker with no illness case. That is why a phased return approach and the gradual reintroduction of duties could lead to a better integration of the patient and an adequate support for maintaining a job longer. Integration in the organization is a first step perspective, followed close by the duration of occupation and job stability.

As mentioned before the phased return approach allows the patient to participate at work in a gradual way, to recover his or her role on the job systematically, to manage his or her own time schedule and tasks, to permit absence if needed for medical reasons and so on. Thus, the patient may feel 
more support from the management team, including here a personalized working scheme and procedures that lead the employee to better adapt and work in the organizational environment he or she is returning to.

Out of the many dilemmas that management faces when dealing with RTW and cancer, one is always present - the sensible subject of confidentiality. In an article Tiedtke et al. (2013, 399-409) observed that managers faced multiple challenges when working with employees on cancer diagnosis. On one hand they have to inform the other co-workers about the condition of their colleague and to communicate with the patient in order to offer support and accommodation, while keeping as much as possible a low profile on the private life of the patient inside the organization; on the other hand managers, in order to provide adequate work conditions and support for the patient, they needed to do so without entering too much in the private life sector of the employee and the list could go on.

Moreover, the confidentiality issue extends also to the point of collecting valuable data inside interviews for research. Many articles state the dilemma which describes the difficult decision upon what is relevant for the researcher and how far can he go with the investigation upon cancer, in the case of both employers and employees that are resilient, based on confidentiality, moral and ethical grounds, to provide answers and information (Nowrouzi et al. 2009; Grunfeld, Low and Cooper 2010; Tiedtke et al. 2013).

Also Tiedtke et al. $(2013,403)$, pointed out the manager's roles in conflict, caught between the decision to hire, maintain and support an employee with cancer and the productivity expectations of the organization, together with the expectations of the other co-workers in terms of equal rights, equal wages, tasks and overcharging with extra-duties in order to help the employee with a special situation. From another point of view, the supervisors/managers perceive themselves as agents of change, protective, problem-solving and key persons in the RTW process (Holmgren and Ivanoff 2007; Aas et al. 2008). 
Despite the good intentions of the decision making actors, the path of integrating employees with cancer diagnosis is difficult now, research information is still lacking, due to a low level of responses and sensible subject profile of the target group. Nowrouzi et al. $(2009,18-20)$ described in their article the numerous barriers that may block the process of RTW or maintaining a job inside the organizations while dealing with a cancer illness throughout this time. Among these, the authors mention (Nowrouzi et al. 2009): the incorrect perception of managers and also co-workers, in some cases, that employees diagnosed with cancer possess more significant limitations at work, than they do in reality; the incorrect perception that patients with cancer cannot manage their job duties simultaneously with illness management; the misperception that this category of subject can work harder than in reality.

Thus, in the present debate, a question resides: is the matter of managing cancer at work only in the hands of decision makers inside the organization? More likely not and, as mentioned before, the dilemma of RTW in the case of patients with cancer lies in a five dimension framework, considering here health professionals and caregivers, employers, human resources experts, researchers and of course employees. Their information upon the subject, their perception on the issue, their level of involvement and degree of collaboration can solve or challenge even more the present topic.

\section{Patient perspective over cancer and RTW}

In managing cancer patients at work, the scholarly literature investigates a variety of themes concerning the patient's perspective on the RTW process and implications.

In this direction a study of Mak et al. $(2014,272)$ sustained the idea that patients who RTW focus on achieving normality at work, a topic described also by Amir et al. (2010). Employees that recovered from a long-term illness and treatment such as cancer or employees that are still undergoing 
treatment or were diagnosed with cancer are all bound to recreate the work and performance conditions before the illness state. Their psychosocial state is sensitive to the need to achieve work results, to fit in the organizational environment and to perform as normal as possible while dealing with the heavy burden of treatment and long-term illness and disability.

From this point of view, in the same article Mak et al. (2014, 272-275) found four possible ways of coping with the cancer dilemma at work in the case of subjects survivors of cancer, evaluated in the study, as follows: adopting an accepting attitude, facing unjust treatment in employment, maintaining a job in order to retain one's ego and power, working harder to meet expectations. The same authors also described other four unintended actions in the same case of subjects: holding a grateful attitude towards the employer to retain the job, manifesting a passive acceptance of perceived discrimination at work, downplaying the illness state to avoid being a burden for the organization and manifesting the fear of losing by compromising the expectations (Mak et al. 2014, 273).

As discussed before in the previous section of the present paper, the collaboration between the management level and employee level is vital for an optimal integration in the organization. Patients with cancer struggle either to re-adapt at work, to provide results as good as they can or to maintain a certain job while following treatment, being exposed daily to changes in their health state, response to treatment, response to work capacity and ability. Experiencing fatigue is one of the most common effects when it comes to patients diagnosed with cancer that are RTW or continuing work during the treatment. The person may manifest fatigue due to the treatment, illness or pressuring working schedule or to the prolonged absence, with no adaptation period when RTW. Kessler et al. (2001) concluded that fatigue, observed as an illness factor, may be associated with a low level in work ability, affecting performance in this manner. Pryce, Munir and Haslam (2007) also noted that fatigue is perceived by subjects as one of the main difficulties when RTW, affecting the work ability. Besides fatigue, other physical factors like nausea or lack of energy alongside 
psychological factors like depression, feeling bored or useless can contribute to low levels in performance and ability at work (Taskila and Lindbohm, 2007; Steiner et al. 2008).

In a study on various target groups, Gudbergsson, Fossa and Dahl (2008) observed that among female subjects who had breast cancer and males who had testicular or prostate cancer, those survivors who changed jobs had lower mental and physical work ability compared to those who did not change jobs. So maintaining the former job, in a well-known work environment, is more likely to be preferred by the patient, in contrast with finding a new job and setting at some point, under the pressure of the unknown and unwanted. From this perspective, the management team has to provide an adequate support strategy and communication scheme in order to avoid social inequalities, discrimination, false expectations and negative coping mechanisms from the patient towards the personal duties, other co-workers or team members and the organization itself. In the same direction, a meta-analysis conducted by De Boer et al. (2009), concluded that cancer survivors faced an increased risk of unemployment, especially survivors of breast cancer, gastrointestinal cancer, and cancer of the female reproductive organs and cancer patients living in countries or times with relatively high unemployment rates. The same authors also sustained the idea that there was a need for the development of interventions where clinicians and other professionals can participate and involve, enhancing cancer patient participation in the work life (De Boer et al. 2009). Vidor et al. $(2014,8)$ supported the idea that interventions from psychologists and physicians specialized in job retention over patients diagnosed with cancer can impact and reduce the social inequalities upon the RTW itinerary.

In another article, O'Baugh et al. $(2003,265)$ concluded that for the general perception of cancer patients, the subjects associated a positive perspective or attitude with the state of a certain normality, this leading to a coping mechanism towards the illness and its treatment.

Also, Park and Shubair $(2013,8-9)$ concluded that RTW itself as a positive process can lead in the case of breast cancer survivors to a better 
healing and control. At the same time, Norredam et al. (2009) observed in another study that female cancer survivors exhibited lower confidence in finding an equally good job in the next few months if they would have lost their actual job, compared to those without cancer. So apart, from other mechanisms, adopting a positive perception or attitude towards the illness associated with the opportunity of RTW, may improve also the patient's level of confidence.

Moreover, employees diagnosed with cancer have the tendency either to accept their special state with resignation, failing to avoid discrimination and personal injustice due to the fear of losing job and status or in another scenario they tend to overpressure their personal energy and abilities in order to keep up with the others, not to show their disability, to fulfil inadequate demands and tasks.

Yet again the situational level and organizational behaviour towards cancer from patient perspective, similar to the case of manager view, is strongly influenced by the existing social policies, laws and procedures, present in the organization's culture and in the society from which the organization resides. For example, in a three year longitudinal study, Maunsell et al. (2004) found little evidence to confirm that in the case of several target groups from Canada, female patients diagnosed with breast cancer, the subjects would have experienced discrimination at work. The authors of the same study also observed that reducing the working hours, changing the activity schedule and especially stopping work was the patient's own decision in most cases (Maunsell et al. 2004).

There is of course a third situation, debated upon in the previous section, in which the patient with the help of others (management, coworkers, family, stakeholders) can integrate gradually in the organization, meet reasonable demands and duties, perform at an optimal level without affecting his or her health state in a negative manner, improving daily the mental and psychological state. 


\section{Conclusions}

With more and more improvements each day inside the medical field of cancer treatment and ultimate generation technology, it may seem that life expectancy and survival rates are increasing rapidly. In this manner, the organizational domain, as well as the research units, have to answer future challenges concerning the re-integration of employees diagnosed with cancer inside a support and discrimination-free structure, in which both employer and employee can reach performance, following organizational goals while maintaining a state of normality and balance for those in need and with special requirements.

From a general organizational perspective, studies showed that, when dealing with the RTW process of cancer patients, the research analysis focuses on different categories of employees such as: former patients that are RTW, patients with cancer that are trying to maintain their work or RTW, patients that are in remission and RTW, patients that fully recovered from cancer but remain with long-term collateral disabilities and are RTW. In this direction, work-related variables, as well as psychosocial variables, should be given a closer attention, due to their implications on research and practice.

Concerning the management sector, the findings in the studies, analysed in the previous sections of the paper, suggest that the introduction of information and positive attitude training of co-workers system can facilitate an easier integration of employees diagnosed with cancer inside the organization. At the same time, from a manager point of view, collaborating with occupational health specialists and managing the working schedule of the patient may lead to a proper adaptation of the employee to the job demands, performance rate and improved personal work abilities.

Moreover, managers have access to a wide range of support measures that can be applied in these cases, among which: control over working hours of the employee, job tasks and deadlines, control over organizational strategies and objectives, distribution of team roles, etc. At the same time, 
the management team has to be informed and trained in order to provide the most adequate working conditions and environment for the employee that is RTW. In other words, research and practice field should focus more on the organizational behaviours which can be shaped and oriented inside the company, as a vivid expression of leaders, workers, partners' attitudes and perceptions towards RTW and cancer. In this light of things, collaboration between management team, occupational health experts, health services and other stakeholders is essential.

In the case of patient perspective over RTW, study findings showed that besides the health variables that the employee faces, the subjects tend to recreate the work and performance conditions before the illness state, reaching for a state of normality at the workplace. Moreover, results concluded that adopting a positive attitude towards the illness increases the chances of job retention and a better integration at work. The employee's behaviour, diagnosed with cancer, will oscillate in different scenarios, from the state of resignation, failing to avoid discrimination and personal injustice to the fear of losing job and status, from the tendency to overpressure their personal energy and abilities in order to keep up with the others to hiding their disability, in order to fulfil inadequate demands and tasks, from reaching the lost work abilities to recovering in a support work climate that values integration and people as a true mobilizing force.

Another conclusion of the study states that in many cases, the findings are very heterogeneous, single element oriented, not following a unitary line, due to the complexity and multi sector field of research, which makes it difficult to draw some general guidelines in the analysis of cancer and RTW mechanisms. In this manner, many questions remain unsolved, among which: how to establish a proper collaboration between management level, health services, employee and stakeholders?, how to measure and analyse the RTW and job retention of cancer patients while respecting their privacy, ethical and moral grounds?, how to ensure an adequate integration of cancer patients when social policies are missing, lack in-depth content or differ so much?, how to ensure adaptation at work while obtaining performance in a 
short time?

The paper underlines the importance of management and patient approaches and perspectives in evaluating the cancer and RTW implications, following several relevant studies. At the same time, the paper triggers a series of questions and draws attention as in the case of other studies that there is a vital need for future research concerning the management sector and the RTW of cancer patients, in the case of those who try to maintain their job or who try to find a new work environment and duties suitable for their condition, state of mind, needs and real expectations.

\section{Acknowledgement:}

This article is based on a research conducted in the project 'Community participation for reducing the burden of cancer: stakeholders' involvement in facilitating the return to work of cancer patients'. The project was supported by a grant from the Romanian National Authority for Scientific Research, CNCS - UEFISCDI [project number PN-II-RU-TE-2014-4-0478].

\section{References:}

Aas, Randi W., Kjersti L. Ellingsen, Preben Lindøe, and Anders Moller. 2008. "Leadership qualities in the return to work process: a content analysis". Journal of Occupational Rehabilitation 18(4): 335-346. DOI 10.1007/s10926-008-9147-y.

Amir, Ziv, Phil Wynn, Fong Chan, David Strauser, Stuart Whitaker, and Karen Luker. 2010. "Return to Work After Cancer in the UK: Attitudes and Experiences of Line Managers". Journal of Occupational Rehabilitation 20(4): 435-442. DOI 10.1007/s10926-009-9197-9.

De Boer, Angela G.E.M., Taina Taskila, Anneli Ojajarvi, Frank J.H. van Dijk, and Jos H.A.M. Verbeek. 2009. "Cancer survivors and unemployment: a meta-analysis". Journal of the American Medical Association 301(7): 753-762. DOI 10.1001/jama.2009.187.

Feuerstein, Michael, Briana L. Todd, Michal C. Moskowitz, Gina L. Bruns, Mallori R. Stoler, Thomas Nassif, and Xinhua Yu. 2010. "Work in cancer survivors: a model for practice and research". Journal of Cancer Survivorship 4(4): 415-437. DOI 10.1007/s11764-010-0154-6. 
Grunfeld, Elizabeth A., Emma Low, and Alethea F. Cooper. 2010. "Cancer survivors' and employers' perceptions of working following cancer treatment". Occupational Medicine 60(8): 611-617. DOI 10.1093/ occmed/kqq143.

Gudbergsson, Saevar Berg, Sophie D. Fossa, and Alv A. Dahl. 2008. "A study of work changes due to cancer in tumor-free primary-treated cancer patients. A NOCWO study". Supportive Care in Cancer 16(10): 11631171. DOI 10.1007/s00520-008-0407-3.

Hoefsmit, Nicole, Angelique de Rijk, and Inge Houkes. 2013. "Work resumption at the price of distrust: a qualitative study on return to work legislation in the Netherlands". BMC Public Health 13(1): 153167. DOI 10.1186/1471-2458-13-153.

Holmgren, Kristina and Synneve D. Ivanoff. 2007. "Supervisors' views on employer responsibility in the return to work process. A focus group study". Journal of Occupational Rehabilitation 17(1): 93-106. DOI 10.1007/s10926-006-9041-4.

Kessler, Ronald C., Paul E. Greenberg, Kristin D. Mickelson, Laurie M. Meneades, and Philip S. Wang. 2001. "The effects of chronic medical conditions on work loss and work cutback". Journal of Occupational and Environmental Medicine 43(3): 218-225. DOI 10.1097/00043764200103000-00009.

Lavigne, Jill E., Jennifer J. Griggs, Xin M. Tu, and Debra J. Lerner. 2008. "Hot flashes, fatigue, treatment exposures and work productivity in breast cancer survivors". Journal of Cancer Survivorship 2(4): 296-302. DOI 10.1007/s11764-008-0072-z.

Maiwald, Karin, Agnes Meershoek, Angelique de Rijk, and Frans J.N. Nijhuis. 2013. "Policy on manager involvement in work reintegration: Managers' experiences in a Canadian setting". Work 49(3): 483-494. DOI 10.3233/WOR-131727.

Mak, Angela Ka Ying, Shirley S. Ho, and Hyo Jung Kim. 2014. "Factors Related to Employers' Intent to Hire, Retain and Accommodate Cancer Survivors: The Singapore Perspective". Journal of Occupational Rehabilitation 24(4): 725-731. DOI 10.1007/s10926-014-9503-z.

Mak, Angela Ka Ying, Suwichit Chaidaroon, Gilbert Fan, and Fahimah Thalib. 2014. "Unintended consequences: the social context of cancer survivors and work". Journal of Cancer Survival 8(2): 269-281. DOI 10.1007/ s11764-013-0330-6.

Maunsell, Elizabeth, Melanie Drolet, Jaques Brisson, Chantal Brisson, Benoit Masse, and Luc Deschenes. 2004. "Work situation after breast cancer: results from a population-based study". Journal of the National Cancer 
Institute 96(24): 1813-1822. DOI 10.1093/jnci/djh335.

McKay, Georgina, Vikki Knott, and Paul Delfabbro. 2013. "Return to work and cancer: the Australian experience". Journal of Occupational Rehabilitation 23(1): 93-105. DOI 10.1007/s10926-012-9386-9.

Munir, Fehmidah, Joanna Yarker, and Hilary McDermott. 2009. "Employment and the common cancers: correlates of work ability during or following cancer treatment". Occupational Medicine 59(6): 381-389. DOI 10.1093/occmed/kqp088.

Norredam, Marie, Ellen Meara, Mary Beth Landrum, Haiden A. Huskamp, and Nancy L. Keating. 2009. "Financial status, employment, and insurance among older cancer survivors". Journal of General Internal Medicine 24(2): 438-445. DOI 10.1007/s11606-009-1034-5.

Nowrouzi, Behdin, Nancy Lightfoot, Kristy Cote, and Rhonda Watson. 2009. "Workplace support for employees with cancer". Current Oncology 16(5): 15-22. http://www.ncbi.nlm.nih.gov/pmc/articles/PMC 2768505/.

O'Baugh, Jenny, Lesley M. Wilkes, Suzanne Luke, and Ajesh George. 2003. "'Being positive': perceptions of patients with cancer and their nurses". Journal of Advanced Nursing 44(3): 262-270. DOI 10.1046/j.1365-2648.2003.02801.x.

Park, Jae-Hyun, Jong-Hyock Park, and Sung-Gyeong Kim. 2009. "Effect of cancer diagnosis on patient employment status: a nationwide longitudinal study in Korea". Psycho-Oncology 18(7): 691-699. DOI 10.1002/pon.1452.

Park, Joanne and Mamdouh Shubair. 2013. "Returning to Work After Breast Cancer: A Critical Review". International Journal of Disability Management 8(1): 1-10. DOI http:/ / dx.doi.org/10.1017/idm.2012.7.

Pryce, Joanna, Fehmidah Munir, and Cheryl Haslam. 2007. "Cancer survivorship and work: symptoms, supervisor response, co-worker disclosure and work adjustment". Journal of Occupational Rehabilitation 17(1): 83-92. DOI 10.1007/s10926-006-9040-5.

Spelten, Evelien. R., Mirjam A. Sprangers, and Jos H. Verbeek. 2002. "Factors related to influence the the return to work of cancer survivors: a literature review". Psycho-Oncology 11(2):124-131. DOI 10.1002/pon. 585.

Steiner, John F., Tia A. Cavender, Deborah S. Main, and Cathy J. Bradley. 2004. "Assessing the impact of cancer on work outcomes: what are the research needs?". Cancer 101(8): 1703-1711. DOI 10.1002/cncr. 20564.

Steiner, John F., Tia A. Cavender, Carolyn T. Nowels, Brenda L. Beaty, Cathy 
J. Bradley, Diane L. Fairclough, and Deborah S. Main. 2008. "The impact of physical and psychosocial factors on work characteristics after cancer". Psycho-Oncology 17(2): 138-147. DOI 10.1002/ pon.1204.

Syse, Astri, Steinar Tretli, and Oystein Kravdal. 2008. "Cancer's impact on employment and earnings-a population-based study from Norway". Journal of Cancer Survivorship 2(3): 149-158. DOI 10.1007/s11764-0080053-2.

Taskila, Taina and Marja-Liisa Lindbohm. 2007. "Factors affecting cancer survivors' employment and work ability". Acta Oncologica 46(4): 446451. DOI 10.1080/02841860701355048.

Taskila, Taina, Rami Martikainen, Paivi Hietanen, and Marja-Liisa Lindbohm. 2007. "Comparative study of work ability between cancer survivors and their referents". European Journal of Cancer 43(5): 914920. DOI 10.1016/j.ejca.2007.01.012.

Tiedtke, Corine, Peter Donceel, Angelique De Rijk, and Bernadette Dierckx De Casterlé. 2013. "Return to Work Following Breast Cancer Treatment: The Employers' Side". Journal of Occupational Rehabilitation 24(3): 399-409. DOI 10.1007/s10926-013-9465-6.

Vidor, Clémence, Ariane Leroyer, Véronique Christophe, Mélanie Seillier, Jérome Foncel, Justine Van de Maële, Jacques Bonneterre, and Sophie Fantoni. 2014. "Decrease social inequalities return-to-work: development and design of a randomised controlled trial among women with breast cancer". BMC Cancer 14(267): 1-10. DOI 10.1186/ 1471-2407-14-267. 